\title{
Archipiélagos coloniales y mexicanos: reimaginando los estudios del Caribe colonial ${ }^{1}$
}

\author{
Yolanda Martínez-San Miguel ${ }^{2}$ \\ University of Miami (Estados Unidos) \\ ORCID ID: 0000-0001-7802-0907
}

Traducción: Rafael Burgos-Mirabal

Recibido: 9 de mayo de 2019

Aceptado: 27 de junio de 2019

\begin{abstract}
RESUMEN
Este ensayo se enfoca en el archipiélago caribeño como una unidad geopolítica e histórica. El archipiélago usualmente combina grupos de islas y sus redes correspondientes de puertos, fortificaciones, plantaciones y ciudades, así como sus sistemas sociales, culturales y de producción. La primera sección analiza un corpus de mapas diseñados por cartógrafos europeos en los que el Caribe se representa como el "archipiélago de México," para meditar sobre cómo los archipiélagos coloniales fueron imaginados en el contexto de la competencia entre imperios de los siglos XVII y XVIII. En la segunda parte de este ensayo, estudio un cuento de Tiphanie Yanique, escritora de las Islas Vírgenes estadounidenses, como un ejemplo de cómo el imaginario archipelágico colonial se transforma en un archipiélago decolonial en la literatura del siglo veintiuno. El ensayo concluye con una reflexión sobre las implicaciones y la utilidad de repensar el Caribe y los estudios caribeños desde una perspectiva archipelágica colonial y decolonial.
\end{abstract}

Palabras clave: archipiélago, Caribe, cartografía, colonial, decolonial, literatura

\begin{abstract}
This chapter focuses on the Caribbean archipelago as a geopolitical and historical unit. An archipelago actually combines groups of islands and their corresponding networks of ports, fortifications, plantations, and cities, as well as their social, cultural, and productive systems. The first section of the chapter analyzes an example from a corpus of maps produced by European cartographers in which the Caribbean is represented as the "Mexican archipelago," to meditate about how colonial archipelagoes were imagined in the multi-imperial competition context of the seventeenth and eighteenth centuries. In the second part of the chapter, a short story by Tiphanie Yanique from the US Virgin Islands provides an example of how this archipelagic colonial imaginary is transformed into a decolonial archipelago in early twenty-first century literature. The chapter closes

\footnotetext{
${ }^{1}$ Colonial and Mexican Archipelagoes: Reimagining Colonial Caribbean Studies

Yolanda Martinez-San Miguel, "Colonial and Mexican Archipelagoes: Reimagining Colonial Caribbean Studies," in Archipelagic American Studies, Brian Roberts, Michelle Stephens, Eds., pp. 155-173. Copyright, 2017, Duke University Press. All rights reserved. Republished by permission of the copyright holder. www.dukeupress.edu

${ }^{2}$ Doctora en Estudios Culturales Latinoamericanos, University of California at Berkeley. Profesora y Marta S. Weeks Chair en Estudios Latinoamericanos, Department of Modern Languages and Literatures, University of Miami. Correo electrónico: ymm34@miami.edu
} 
with reflections on the implications and utility of rethinking the Caribbean and Caribbean studies from a colonial and decolonial archipelagic perspective.

Keywords: archipelago, Caribbean, cartography, colonial, decolonial, literature

\section{INTRODUCCIÓN}

Este ensayo constituye una reflexión acerca de la intersección de los estudios coloniales e imperiales que se enfoca en el archipiélago del Caribe como unidad geopolítica e histórica. Pienso el archipiélago tanto en cuanto a marco conceptual como en cuanto a conceptualización geográfica. Al historizar así tal unidad, se posibilita una redefinición de los estudios caribeños que favorezca el establecimiento de conexiones con diversas regiones que comparten contextos coloniales/ imperiales semejantes, como las Filipinas, las Islas Canarias, las Islas Azores y Hawai'i, entre otras. Un enfoque específico sobre regiones coloniales-archipelágicas me permite analizar cómo los conjuntos de islas tienden a concebirse mediante estructuras administrativas e imaginarios simbólicos que se alejan de los paradigmas continentales y de soberanía dominantes en los marcos conceptuales de muchos estudios regionales o de área.

Al definir los archipiélagos como sistemas de islas, y el mar que los vincula, mi trabajo se nutre del campo de los estudios insulares, aunque sin coincidir del todo con los principales planteamientos que en este campo se suelen atender. El estudio de islas se fija en los países y en las regiones cuya organización geográfica y geopolítica es insular. De ahí que el análisis de los estudios insulares se haya centrado en "la constitución de la 'insularidad' y sus posibles o plausibles influencias e impacto en la ecología, la conducta humana y de otras especies, y en cualquiera de las áreas investigadas por materias tradicionales monodisciplinares (como arqueología, ciencias económicas o literatura), por materias multidisciplinares (como economía política o biogeografía) o por enfoques y/o temas de política pública (como gobierno, capital social, eliminación de residuos, extinción de lenguas o turismo sostenible)" (Baldacchino, 2006: 9). En épocas más recientes, otra línea de investigación de los estudios insulares se ha enfocado en las conexiones archipelágicas entre las islas, así como los vínculos de las islas con las regiones continentales (Pugh, 2013; Stratford, et al., 2011). Con el fin de complejizar nuestra conceptualización del Caribe como parte de una conversación más amplia con y sobre otras regiones que comparten un conjunto de condiciones similares, mi definición de archipiélago incluye grupos de islas, con sus correspondientes redes de puertos, fortificaciones, sistemas de plantaciones y ciudades, así como con sus sistemas sociales, culturales y de producción.

El concepto de archipiélago no es nuevo en los estudios caribeños. El Caribe se ha concebido como un sistema de islas que entraña una identidad colectiva -como en la designación de "las Antillas" o la "Federación de las Indias Occidentales"-, o una experiencia política común, caracterizada por la fragmentación y por la ausencia de una noción simple de orden -como la "frontera imperial" que propuso Juan Bosch (1981). Algunos países constan de más de una isla como entidad nacional unitaria, como es el caso de Trinidad y Tobago. Muchos caribeñistas cuestionan la definición 
de la región como predominantemente insular. En mi caso, no conceptualizo los archipiélagos como exclusivamente insulares, sino que incluyo en el Caribe zonas continentales en un contexto multi-focal en donde se alternan islas con regiones costeras conectadas.

Los taínos se organizaron en cacicazgos en el Caribe, estableciendo redes de comercio y de intercambio con más de una isla y, en ocasiones, vincularon a ciertas islas con territorios en la tierra firme (Keegan and Maclachlan, 1989). Así pues, aun en las articulaciones indígenas del espacio, los archipiélagos -aunque no necesariamente las islas por separado- funcionaban como unidades fluidas de pensamiento, de conocimiento o de identificación. De igual modo, se encuentran modos de un pensar "archipelágico" desarrollado en el trabajo de algunos investigadores y pensadores caribeños fundacionales (Benítez-Rojo, 1996; Glissant, 1997).

Mi trabajo aspira a expandir las ideas de investigadores y pensadores fundacionales que han revivido conceptualizaciones del Caribe como un sistema de islas. Complemento su aporte fundamental estableciendo un diálogo con estudios más recientes que se refieren a la región como un "archipiélago imperial" (Morillo Alicea, 2005; Thompson, 2010) o como un "paradigma inter-atlántico" de colonización (Stevens-Arroyo, 1993). En estos estudios, el archipiélago se utiliza para analizar cómo las condiciones geográficas han tenido un impacto en el proceso histórico de incorporación imperial de ciertas regiones durante los siglos XVI a XIX, como en los casos de las Islas Canarias, del Caribe y de las Filipinas. Por ejemplo, Lanny Thompson ha reflexionado sobre cómo los Estados Unidos produjeron discursos diversos para asimilar a sujetos "otros", por medio de la insularidad colonial (Thompson, 2010). Otros críticos e historiadores culturales han aportado estudios en que vinculan varias posesiones insulares estadounidenses, tales como el vínculo entre las Filipinas y Cuba durante la segunda mitad del siglo XIX (Blanco, 2009; Hagimoto, 2013; Rafael, 2005). También Elizabeth M. DeLoughrey ha realizado estudios comparativos de las literaturas producidas en las islas del Pacífico y del Caribe, dándole seguimiento a los puntos de contacto —incluyendo tanto aspectos en común como diferencias - que han constituido los imaginarios insulares y archipelágicos en estas dos regiones (DeLoughrey, 2010).

Los acercamientos culturales a los estudios archipelágicos y del Caribe podrían interrogar la función del archipiélago en la articulación simbólica de una conceptualización más amplia de esta región. Decididamente, hay mucho que aprovechar de los trabajos orientados a una sola isla, como las reflexiones de Antonio S. Pedreira sobre Puerto Rico en su libro-ensayo Insularismo de 1934 y las reflexiones sobre Antigua de Jamaica Kincaid en A small place (1988). ${ }^{3}$ No obstante, los estudios caribeños necesitan superar el modelo insular como marco central debido, precisamente, a que ese paradigma no nos permite enfocarnos en las diversas maneras en que los archipiélagos, y no las islas, han funcionado como la unidad de análisis que facilita la concepción de ciertas regiones coloniales.

\footnotetext{
${ }^{3}$ En Insularismo, Antonio S. Pedreira propone dar respuesta a la cuestión de la identidad nacional de Puerto Rico en la década de 1930 . Al hacer esto repara en la condición colonial de la isla y argumenta que el insularismo -la condición de aislamiento o de isleñismo- es la causa por la cual el país no ha podido ser nunca un estado soberano ni ha logrado proyectarse más firmemente en el plano histórico-político (Pedreira, 1992 [1934]). En A small place, Jamaica Kincaid reflexiona acerca de las dinámicas psicológicas asociadas con escribir desde una pequeña isla del archipiélago caribeño (Kincaid, 1988).
} 
Siguiendo el importante trabajo de Elaine Stratford, Godfrey Baldacchino y Jonathan Pugh -quienes ponen de relieve las ventajas del marco de archipiélago en lugar del insular (Baldacchino, 2006; Pugh, 2013; Stratford et al., 2011)- nuestras definiciones del Caribe necesitan incluir no solo sus islas y archipiélagos sino también sus particulares relaciones histórico-políticas con el colonialismo. Esto resulta especialmente pertinente en el caso de las regiones del Caribe que experimentaron lo que en otro lugar he denominado como el colonialismo extendido, que

[...] comenzó en los siglos XVI y XVII, y se extendió hasta el siglo XX (y en algunos casos se extiende hasta el día de hoy), y [que] incluye con frecuencia la coexistencia de más de un sistema colonial (el español y el francés en el caso de Martinica; el español y el estadounidense en Cuba, Puerto Rico y las Filipinas; el español, el francés y el inglés en muchas islas del Caribe anglófono) (Martínez-San Miguel, 2014)

Muchas regiones que han experimentado más de un régimen colonial a lo largo de un periodo prolongado de tiempo también se han concebido como sistemas de espacios insulares distintos de los espacios soberanos continentales (Stratford et al, 2011). Y, como resultado, los archipiélagos coloniales tienden a presentar lo que muchos consideran "disposiciones únicas de estatus dentro de cuerpos nacionales o supranacionales mucho más amplios" (Baldacchino, 2006). Por mi parte, argumento que tales relaciones atípicas con la soberanía y el nacionalismo son el legado de su inusitada condición como posesiones imperiales de ultramar. ${ }^{4}$

En la siguiente sección discuto un ejemplo sacado de un corpus de mapas producidos por cartógrafos europeos, en los cuales se representa al Caribe como el "Archipiélago mexicano". Este ejemplo me permite realizar un análisis acerca de cómo se imaginaban los archipiélagos imperiales y coloniales en el contexto de la competencia entre imperios de los siglos XVII y XVIII, época de transición de la era mercantil europea al sistema-mundo del capitalismo mundial (Wallerstein, 1974). Más adelante analizaré también el Caribe poscolonial de nuestros días usando el ejemplo que ofrece Tiphanie Yanique en uno de sus relatos. Yanique es una escritora contemporánea caribeña de las Islas Vírgenes estadounidenses. Su cuento ilustra cómo se transforma el imaginario colonial de archipiélago en un archipiélago descolonial a principios del siglo XXI. El estudio de estos casos permite considerar nuevas reflexiones tanto sobre las implicaciones como sobre la utilidad de repensar el Caribe utilizando el concepto de archipiélago colonial.

\section{El Archipiélago de México: "un plan para la colonización"5}

La dimensión simbólica de los mapas también los conecta con otros textos. Los cartógrafos modernos por lo general consideran sus mapas como declaraciones de hechos escritas en el lenguaje de las matemáticas, pero son siempre metáforas o símbolos del mundo.

J.B. Harley. "Textos y contextos en la interpretación de mapas antiguos" (2001: 37)

\footnotetext{
${ }^{4}$ Yarimar Bonilla estudia la problemática relación entre soberanía y la formación de los estados caribeños (Bonilla, ${ }^{2012}$ ).

${ }^{5}$ Tomo esta frase de Patricia Mohammed (Mohammed, ${ }^{2009}{ }^{72}$ ).
} 
Los mapas son documentos históricos y simbólicos densos que pueden enriquecer nuestro estudio del colonialismo, el postcolonialismo y la descolonización en el Caribe. El trabajo fundacional de J. B. Harley es especialmente iluminador sobre esta cuestión. Su pensamiento revela las dimensiones simbólicas, metafóricas y de ficción que poseen los mapas, abriendo así la posibilidad para que se puedan realizar lecturas minuciosas de estos textos para analizar sus intenciones, funciones y perspectivas. Según Harley, los mapas son documentos históricos y culturales, que deben leerse simultáneamente tanto por la información que en ellos se destaca como por lo que se le resta importancia o aun se silencia (2001: 45). De igual modo, propone que los mapas constituyen un sistema de comunicación tan complejo como un idioma y utiliza la crítica literaria para definir el "discurso" cartográfico, concepto medular en su ensayo fundacional "Mapas, saber y poder" (pp. 54-55).

Otro elemento central para reflexionar sobre el Caribe como un sistema archipelágico colonial es el análisis meticuloso de los vínculos entre la cartografía y el colonialismo europeo, conforme este se propagaba en las Américas (Mundy, 1996; Padrón, 2004) y específicamente en el Caribe (Arias, 2010; Mohammed, 2009). Ricardo Padrón usa la noción de "literatura cartográfica" para acercarse a las representaciones iconográficas y discursivas del espacio (en mapas, relaciones) y a textos literarios relacionados con las concepciones del imperio español durante la temprana modernidad (Padrón, 2004: 45). En este ensayo expando el ámbito de utilidad de la noción de literatura cartográfica como marco conceptual para estudiar las representaciones cartográficas y literarias del Caribe desde la década de 1650 hasta el presente, con el fin de recuperar los motivos históricos e ideológicos que articulan las representaciones -tanto de la temprana modernidad como las contemporáneas- de esas posesiones archipelágicas.

Concierne especialmente a este proyecto un pequeño corpus de mapas producidos por cartógrafos europeos entre 1650 y 1750 . En estos, el Caribe se concibe como el "archipiélago mexicano". ${ }^{5}$ Este es el mismo periodo en que Francia e Inglaterra libraron lo que Patricia Mohammed ha descrito como una "guerra de mapas", una serie de conflictos durante los cuales "[...] se utilizaban los mapas entre las potencias europeas rivales para reclamar territorios en el Nuevo Mundo" (Mohammed, 2009: 67). Un mapa creado en 1689 sirve de punto de partida para esta reflexión, como ejemplo de cómo los países europeos en contienda constituían al Caribe como un "archipiélago colonial", conforme intentaban expandir su control sobre los territorios de ultramar en las Américas.

\footnotetext{
${ }^{6}$ Hasta inicios del siglo XVIII, los españoles consideraban que sus mapas políticos, comerciales y náuticos eran documentos secretos solo para uso interno (Harley, ${ }^{2001}:{ }^{59}$; Mohammed, ${ }^{2009:}{ }^{62} \mathrm{y}^{67}$ ). Por esta razón, analizo cómo los cartógrafos no españoles imaginaban y representaban las posesiones españolas y otras posesiones europeas. Esta demarcación nacional no es tan clara como parecería debido a que algunos cartógrafos europeos no españoles también prestaban sus servicios a la corona española.
} 


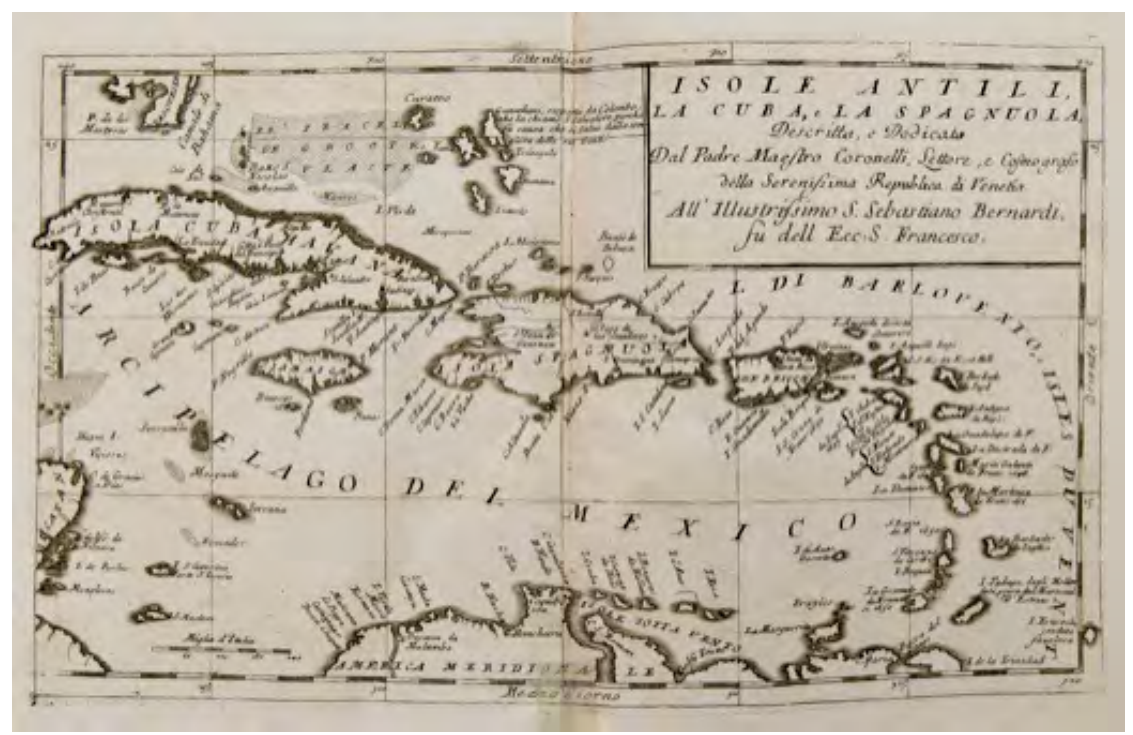

Mapa "Isole Antili, La Cuba e la Spagnuola". En Vincenzo Coronelli. Citta, Fortezze, Isole e Porti Principali dell'Europa. Venetia: Domenico Padouani, 1689. Reproducción cortesía de Newberry Library, Chicago. \#Ayer 135.C8.1689, Plate 95.

En este mapa, se identifica el Caribe entero como el "Arcipelago del Mexico". En el mismo libro donde se incluye este mapa, Vincenzo Coronelli ${ }^{7}$ identifica también algunas de estas islas por separado -Cuba, La Hispaniola y Jamaica- como parte del Archipiélago mexicano, lo que implica que el término se refiere tanto a las islas como al mar que las rodea. Lo que es importante sobre este grupo particular de mapas es que la designación de archipiélago se constituye visual y geopolíticamente como unidad singular que identifica la relación imperial entre los centros metropolitanos y las posesiones ultramarinas que se conciben como una red de islas.

Al margen de los diversos mapas en que la región se representa como el "Mar mexicano" y las islas como el "archipiélago mexicano", hay referencias por escrito más o menos coetáneas en que se presenta al archipiélago de México como un dato que formaba parte del conocimiento general. Este es el caso del libro de Herman Moll titulado Sistema de geografía; o nueva y exacta descripción de la Tierra

Islas de las Antillas.

Después de haber hecho una descripción específica de las diversas partes del continente de la América del Norte, junto a algunas de las islas principales que yacen frente a sus costas; es requisito de igual guisa describir la sexta y última parte de la misma América, lo cual quiere decir, las Islas del Archipélago de México. Son llamadas comúnmente, por diversos geógrafos, Antillae q.d. Ante Insulae, i.e. Las Islas Anteriores, por razón de su situación antes del golfo de México, y en cuanto a que quienes zarpan de Europa, o de África, son las primeras que se avistan antes de la Costa de la Nueva España: otros solo conocen por ese nombre a las cuatro islas mayores, viz. Cuba, Jamaica, La Española y Puerto Rico; y hay quienes agregan las Islas Caribe. Pero aquí adoptaremos el término de Las Antillas en su sentido más amplio, y comprendiendo así a todas las islas que están entre La Florida,

\footnotetext{
${ }^{7}$ Vincenzo Coronelli (1650-1718) fue un monje franciscano nacido en Venecia. Era conocido como cartógrafo, cosmógrafo y editor. A finales de los 1680 fue invitado a París para que construyera globos terráqueos para Luis XIV, Rey de Francia. Años después, ocupó el cargo de cosmógrafo de Venecia. Ver el trabajo de James Lawrence Fuchs sobre Coronelli (Fuchs, 1983).
} 
La descripción de Moll acerca del Caribe es significativa porque representa a las Antillas como una región específica que de hecho constituía el primer punto de contacto y de entrada hacia las Américas. Moll concebía a las Antillas como un sistema, y en este pasaje la idea del archipiélago funciona a manera de categoría de trabajo para referirse al conjunto de islas situadas entre América del Norte y América del Sur. Esta conceptualización de las Antillas evoca también la etimología de archipelago (en inglés) de principios del siglo XVI como chief sea ("archi-mar" o "mar principal" ("Archipelago"). En este caso, sin embargo, Moll se refiere a la región mexicana, y aun quizás [tácitamente] compara al golfo de México con el mar Egeo, y a las islas del archipiélago mexicano con las islas del Egeo.

Esta referencia al México-archipiélago se convierte en moneda corriente a tal punto que se incluye en la definición de archipelago en Le grand dictionnaire historique de Moréri, de 1707, aunque se utiliza para definir al golfo de México en lugar de a las islas como tales: "El ARCHIPIÉLAGO DE MÉXICO, es propiamente el golfo de México, en donde hay muchas islas" (Moréri, 1707). Esta entrada de diccionario es reveladora, porque las islas del Caribe se hacen invisibles con el fin de favorecer su ubicación en el golfo de México, conforme se asocian más con el Virreinato de la Nueva España que con alguna región particular del imperio español.

Por último, encontramos otra vez el término en el siglo XIX, en el Diccionario fraseológico real: inglés-francés (1853). En este caso se confunden en una la definición de isla con la de las Indias Occidentales: "ISLA, fem. sing. island. Las islas, las Indias Occidentales, las islas del archipiélago de México" (Tarver, 1853). En este contexto, el archipiélago del Caribe se ha convertido en sinónimo de Indias Occidentales, y el Caribe en el de territorios coloniales de ultramar de diversos países europeos. Dicho esto, mi interés en esta descripción geográfica intenta responder a dos preguntas. La primera: ¿por qué se concibe el Caribe como una dependencia administrativa, política y aun económica de Mexico? La segunda: ¿por qué el concepto de archipiélago -como sistema de islas, de puertos y de centros de producción, junto al mar que los conecta- es significativo en este contexto en particular?

La respuesta a la primera pregunta requiere una contextualización que deberá realizarse a través de diversos estratos administrativos, económicos y políticos. En primer lugar, deberemos repasar la estructura administrativa del imperio español en las Américas. En España, el Rey administraba las posesiones americanas. La Casa de Contratación, que se funda en 1503, reglamenta el comercio y

\footnotetext{
${ }^{8}$ Antilles Islands.

After having made a distinct Description of the several Parts of the Continent of Northern America, together with some of the principal Islands that lye over against them; it is requisite in like manner to describe the sixth and last Part of the same America, that is to say, the Islands of the Archipelago of Mexico. They are all in general call'd by divers Geographers Antillae, q. d. Ante Insulae, i.e. The Fore-Islands, by reason to their Situation before the Gulph of Mexico, and in regard that they first come in sight to those that sail from Europe, or Africa, before the Coast of New Spain: Others only understand by that Name, the four greater Islands, viz. Cuba, Jamaica, Hispaniola, and Porto Rico; to which some add the Caribbee-Isles. But we shall here take the Term Antilles in its largest Signification, comprehending all the Islands which appear between Florida, a Part of Northern America, on the North; and Terra Firma, a Province of Southern America, on the South (Moll, 1701: 183).

9 “ARCHIPELAGUE DE MEXIQUE, est proprement le golfe de Mèxique, où il a de plusieurs Isles" (Moréri, 1707).

10 "ÎLE, s. f. island. Les îles, West Indies, the islands of the archipelago of Mexico" (Tarver, 1853).
} 
el intercambio de recursos y de bienes, así como las rutas de navegación." El Consejo de Indias -fundado en 1524- se hacía cargo de la administración jurídica y política de las colonias y nombraba a los funcionarios imperiales. En las Américas, las unidades administrativas superiores eran los virreinatos: de la Nueva España, del Perú, de la Nueva Granada y de la Plata; seguidas por las audiencias regionales -como la de Santo Domingo- y las capitanías generales locales: Santo Domingo, 1535; las Filipinas, 1574; Puerto Rico, 1580; Cuba, 1607; Guatemala, 1609 y Yucatán, 1617. De acuerdo con esta estructura, el Caribe, como hoy día lo pensamos, quedaba claramente supeditado en lo administrativo al Virreinato de la Nueva España. Aun así, es importante advertir que el Caribe insular, o México archipelágico, fue el primer territorio colonial del imperio español. Además, las fronteras administrativas entre los virreinatos y las audiencias no estaban deslindadas tan claramente. Las Audiencias Generales se encargaban de la administración jurídica de la región y respondían directamente al Rey de España, y por tal razón, Santo Domingo ocupaba un lugar administrativo ambiguo como sede del primer tribunal de la Corona española en las Américas. Debido a que el presidente de la Audiencia de Santo Domingo también era el Capitán General, este funcionario colonial gozaba de amplias facultades y autonomía para administrar las posesiones españolas en el Caribe (Lockhart y Schwartz, 1989: 102-106).

La situación era aún más compleja debido a que Cuba fue el punto de partida de la expedición original a lo que más adelante se conocería como el Virreinato de la Nueva España y continuó siendo puerto estratégico para diversas rutas de navegación del imperio español. Por ello, su ubicación en la estructura de poder de la administración imperial era ambigua cuando menos. Posiblemente, la ruta de navegación más importante era el sistema de flotas, la cual cobró gran importancia durante los siglos XVII y XVIII, durante el apogeo de la pugna imperial por las posesiones americanas mediante la piratería y el contrabando. El sistema de flotas funcionó entre 1566 y 1790 y consistía en un convoy que se utilizaba para proteger a las embarcaciones que transportaban plata, oro, productos agrícolas y bienes exóticos o costosos, tanto hacia los pocos puertos autorizados, como desde los mismos, para realizar las funciones del comercio oficial entre el Pacífico, el Nuevo Mundo y España. Las flotas navegaban dos rutas principales; la más importante, la Flota de Indias, salía de la Casa de Contratación, en Sevilla y llegaba a los puertos continentales de Veracruz, Portobelo y Cartagena. Luego, las naves se reunían en La Habana, antes de regresar a España. ${ }^{12}$ La otra flota, secundaria, era el Galeón de Manila, la ruta comercial que entre 1565 y 1815 vinculaba a Europa con el codiciado Oriente, y que traía plata de las Américas al Pacífico y transportaba seda, especias exóticas y otros productos de China a través de una ruta vía Manila, Acapulco, Veracruz, el Caribe, las Islas Canarias y Europa. Las mercancías que venían de Manila llegaban a Acapulco y eran transportadas por tierra hasta Veracruz, en donde se unían a la Flota de Indias para regresar a España. En el contexto de estas rutas comerciales, resultan evidentes la competencia y la centralidad alternativa

\footnotetext{
${ }^{11}$ La Casa de Contratación supervisaba las misiones de exploración españolas y en ella quedaban archivados los documentos de descubrimientos con carácter secreto. Uno de esos documentos era el Padrón Real, un mapa máster del mundo que constantemente actualizaban cartógrafos expertos (Harley, 2001: 93).

${ }^{12}$ Ver los trabajos de Curtis Nettels y de Timothy R. Walton sobre las rutas del sistema de flotas (Nettels, 1931; Walton, 1994).
} 
del Virreinato de la Nueva España y La Habana. ${ }^{13} \mathrm{Al}$ mismo tiempo, las rutas de estos dos sistemas de flotas vinculaban entre sí a una serie de archipiélagos coloniales que eran administrados a través del Virreinato de la Nueva España.

Desde el punto de vista financiero, sin embargo, el Virreinato de la Nueva España funcionaba como las arcas reales. Por tal razón, la Nueva España constituía el centro económico de hecho del imperio español y, como tal, facilitaba la administración de las posesiones del Pacífico y del Atlántico. La Nueva España constituía una ubicación estratégica para los galeones de Manila. El Virreinato de la Nueva España era también el centro administrativo del situado mexicano, una subvención que enviaba la Corona a Puerto Rico y a las Filipinas, y que se utilizó entre 1587 y el siglo XIX, entre otras cosas, para construir el sistema de fortificaciones de defensa en el Caribe insular y continental, que incluía Cuba, La Hispaniola, Puerto Rico, Cartagena, Venezuela, Panamá y Veracruz (González Vales, 2016).

En este contexto, los mapas que los cartógrafos europeos produjeron se referían, en pocas palabras, al papel central que el virreinato de Nueva España desempeñaba en la administración de los territorios insulares. El Virreinato gozaba de una completa centralidad en la encrucijada histórica de competencia imperial europea que se desencadenó en la región, y que más adelante balcanizará al archipiélago mexicano en divisiones políticas, culturales y lingüísticas que aun hoy día se conocen y se viven en el Caribe. La estructura administrativa imperial, centralizada en la Nueva España, ha suscitado conceptualizaciones recientes, más complejas y enriquecedoras, como la del Gran México, definido por Américo Paredes como “...todas las zonas habitadas por personas de cultura mexicana -no solamente dentro de los límites de la república de México sino también en los Estados Unidosen sentido cultural y no político" (Paredes, 1976: xiv). ${ }^{8}$ En am ${ }_{-}^{14}$ os casos, la Nueva España/México sirve como una instancia mediadora o frontera activa entre centros metropolitanos (España/los Estados Unidos) y periferias coloniales. El trabajo de José David Saldívar sobre el Gran México también nos recuerda las rutas del Asia y del Pacífico que pasaba por México, y muestra la centralidad más contemporánea de los archipiélagos en los imaginarios culturales mexicano y chicano/ mexicoamericanos (Saldívar, 2012).

Bernardo de Balbuena, en su famoso poema La grandeza mexicana (1603) prefigura la centralidad financiera del Virreinato de la Nueva España, que luego se traducirá en predominio administrativo y político (Balbuena, 1971 [1603]). Barbara Fuchs y yo, en una lectura pormenorizada de este poema, hemos argumentado que "[...] en La grandeza mexicana, Balbuena produce un nuevo imaginario que desplaza el centro del imperio español hacia el virreinato de Nueva España, al concebirlo desde una ciudad de México que funciona como bisagra y centro de operaciones entre los mercados europeos y asiáticos" (Fuchs y Martínez-San Miguel, 2009: 677). En semejante dirección, Gunder Frank sostiene que los Galeones de Manila movilizaron el primer circuito comercial realmente de orden mundial (Frank, 1998) y, en este contexto, la Nueva España funcionaba como

\footnotetext{
${ }_{13}^{13}$ Para más información sobre los Galeones de Manila, ver las investigaciones de Katharine Bjork, de Dennis O. Flynn y Arturo Giráldez y la de William Schurz (Bjork, 1998: Flynn y Giráldez, 1995; Schurtz, 1959).

${ }^{14}$ El concepto del Gran México es también central en José Limón (Limón, 1998) y en José David Saldívar (Saldívar, 2012).
} 
lo que Barbara Fuchs y yo hemos llamado una "metrópoli colonial" (Fuchs y Martínez-San Miguel, 2009: 677-78). Por lo tanto, al referirse al Caribe como el "Archipiélago mexicano", estos cartógrafos europeos reconocían la preponderancia financiera y comercial de hecho de la que gozaba la Nueva España por encima de la importancia militar y náutica de las Capitanías Generales de Cuba y de Santo Domingo.

Aún queda por responder a la segunda pregunta: ¿por qué es el archipiélago la categoría significativa para referirnos a esas posesiones coloniales de ultramar? Una respuesta a esta pregunta aflora de unos pocos textos coloniales en que el Caribe funciona como referente espacial central. El archipiélago colonial/imperial ha funcionado como elemento principal en el caso de narrativas que se han producido en y acerca del Caribe, desde el periodo colonial hasta el presente. Por ejemplo, aunque Colón pensaba que llegaba a otro archipiélago (Cipango/Japón), ${ }^{15}$ el hecho es que su "descubrimiento" de las Américas estaba primero mediatizado por la naturaleza geopolítica del archipiélago caribeño como una red de islas que literalmente desplazó y pospuso la muy deseada ruta hacia el Oriente.

El archipiélago como sistema potencial de territorios, o como frontera, es un tema recurrente e importante en muchos textos coloniales. Solo hay que recordar las descripciones de Las Casas sobre la "destrucción" y "despoblación" de las Indias, en las cuales las islas son más vulnerables a la brutal aniquilación de las poblaciones indígenas que las posesiones de la tierra firme. La repetición de este modelo de destrucción es tan frecuente en el texto que Las Casas crea un esquema explicativo que se aplicó a múltiples posesiones insulares en las Américas. Cito este pasaje como ejemplo:

\section{DE LAS DOS ISLAS DE SANT JUAN Y JAMAICA}

Pasaron a la isla de Sant Juan y a la de Jamaica (que eran unas huertas y unas colmenas) el año de mil y quinientos y nueve los españoles, con el fin y propósito que fueron a la Española. Los cuales hicieron y cometieron los grandes insultos y pecados susodichos, y añadieron muchas señaladas y grandísimas crueldades más, matando y quemando y asando y echando a perros bravos, y después oprimiendo y atormentando y vejando en las minas y en los otros trabajos, hasta consumir y acabar todos aquellos infelices inocentes: que había en las dichas dos islas más de seiscientas mil ánimas, y creo que más de un cuento, y no hay hoy en cada una doscientas personas, todas perecidas sin fe y sin sacramentos (Las Casas, 1988 [1552]: 86).

En algunos casos, este mismo motivo geopolítico ha permitido a ciertos autores producir narrativas "de archipiélago", en las cuales la imbricación de islas y personajes es más central que la articulación de cierta identidad imperial o nacional, como es el caso de la Narrative of a Voyage to the West Indies and Mexico, 1599-1602 de Samuel Champlain. ${ }^{16}$ Champlain viaja a las Indias

\footnotetext{
${ }^{15}$ Colón escribió: "Qusiera oy partir para la isla de Cuba, que creo que debe ser Cipango, según las señas que dan esta gente de la grandeza della y riqueza" [entrada para el martes, 23 de octubre, pág. 42]. Colón también menciona Cipango en sus entradas para el sábado, 6 de octubre y para el martes, 23 de octubre, respectivamente (Colón, 1492: 18 y 424).

${ }^{16}$ Samuel Champlain (1574-1635) fue navegante, cartógrafo, geógrafo, cronista, explorador, colonizador y diplomático francés. En Canadá se le considera como una figura histórica célebre por haber hecho el primer mapa de la costa. Fue fundador de la ciudad de Quebec y es conocido como el que consolidó "las colonias francesas en el Nuevo Mundo". Su Brief Discours des Choses plus remarquables que Sammuel Champlain de Brouage a reconneues aux Indes Occidentalles au voiage qu'il en a faict en icettes en l'année 1599 et en l'année 1601, comme ensuite no se publicó en francés sino hasta 1870 y la primera edición en inglés se publicó en 1859 (Ritchie, 2014).
} 
Occidentales para lograr el acceso a información que de otra forma no estaría al alcance de los franceses. Su narración vincula a la región del Caribe con la Nueva España. El viaje se representa como un crescendo que culmina con la descripción hiperbólica y superlativa de las riquezas de la ciudad de México (Champlain, 1859). Evidentemente, las islas del Caribe y sus puertos no son tan ricos como el virreinato mexicano, aunque Champlain encuentra en esas regiones periféricas buenos puertos en que los mercaderes ingleses y franceses podían intercambiar bienes como parte del mercado mundial que opera al margen de los controles mercantiles del imperio español. Además, en esta narrativa, los archipiélagos funcionan como una constelación o red de navegación que hace posible el viaje entre Europa y la Nueva España:

Al salir de las Islas Canarias, pasamos el golfo de Las Damas, teniendo el viento a popa, de modo que en dos meses y seis días de haber zarpado de San Lúcar, avistamos la isla llamada La Deseada, que es la primera isla que los pilotos necesariamente deben reconocer para dirigirse a todas las demás islas y puertos de las Indias.

Estuvimos cuatro meses en La Habana, y saliendo de esta con toda la flota de las Indias, que allí se había reunido de todas partes, nos dirigimos a pasar el canal de Bahan (Bahama), que es el pasaje de consecuencia, y el cual es necesario atravesar al regresar de las Indias.

Cuatro días después de pasar la Bermuda enfrentamos tan recia tempestad que todo nuestro armamento estuvo seis días sin poder conservarse junto. Después de que pasaron los seis días, medró el tiempo y la mar amainó, nos reunimos todos, y tuvimos viento favorable hasta apercebir las Azores [...] Todas las embarcaciones que regresaban de las Indias por necesidad han de llegar a las mentadas islas de las Azores para hacer sus observaciones, pues de lo contrario, no con certeza podrán acabar su ruta (Champlain, 1859: 6, 45, 48; las cursivas son mías). ${ }^{17}$

Así, en la narrativa de Champlain, los archipiélagos se transforman de una categoría geopolítica en una estructura de navegación y una estrategia discursiva con que se vinculan Europa y las Américas. Al mismo tiempo, los archipiélagos pasan a formar parte de la narrativa para representar las vulnerabilidades de los modelos colonizadores y explotadores que aplicaron los españoles (Las Casas, Champlain), o funcionan como un escenario repetitivo que desplaza y pospone el arribo al codiciado oriente (Colón) o a la riqueza superlativa de la Nueva España (Champlain). Por último, si hacemos dialogar el mapa que analizamos al inicio de este apartado con las narrativas coloniales que acabamos de comentar, los archipiélagos parecen apuntar simbólicamente a la tensión entre

\footnotetext{
17 "Leaving the Canary Islands, we passed by the Gulf of Las Damas, having the wind astern, so that in two months and six days after our departure from St. Lucar, we got sight of an island named La Deseade, which is the first island that pilots must necessarily recognize in going to all the other islands and ports of the Indies. [...]

We were four months at the Havanna, and leaving it with the whole fleet of the Indies, which had assembled there from all parts, we proceeded to pass the channel of Bahan (Bahama), which is the passage of consequence, and which must necessarily be passed in returning from the Indies. [...] Four days after passing Bermuda we had such a great tempest, that the whole of our armament was more than six days without being able to keep together. After the six days had passed, the weather becoming finer and the sea more tranquil, we all reassembled, and had the wind favourable till we perceived the Açores. [...] All vessels returning from the Indies must of necessity approach the said islands of Açores to take their observations, otherwise, they could not with surety finish their route" (Champlain, 1859: 6, 45, 48; las cursivas son mías).
} 
economías imperiales (España, el Virreinato de la Nueva España) y las fronteras coloniales (las Islas Canarias, el Caribe insular y las Filipinas).

\section{III. "Nuestras nuevas posesiones": el archipiélago decolonial como poética}

Los legados y las transformaciones del archipiélago imperial/colonial se pueden detectar en imaginarios caribeños decoloniales más recientes. Este es el caso del trabajo de la escritora contemporánea Tiphanie Yanique, oriunda de un archipiélago que hoy día es uno de los territorios no incorporados de los Estados Unidos. Aunque Yanique parece escribir en un contexto muy distinto del del archipiélago mexicano -es decir, no desde el contexto hispanófono sino del anglófono, ni el de la era colonial sino la época actual, ni en relación con un imperio del Viejo Mundo sino con el imperio estadounidense-, el tratamiento en su trabajo de las Islas Vírgenes de los Estados Unidos puede leerse desde el marco del archipiélago colonial. Aunque de primera instancia la conexión entre el trabajo de Yanique y los mapas y textos de la era colonial que acabo de comentar podría parecer un tanto tenue, el imaginario del archipiélago mexicano continúa siendo una referencia importante en este caso. Tiphanie Yanique es una escritora caribeña diaspórica. Nació en las Islas Vírgenes de los EE.UU. y en el presente vive en Brooklyn, Nueva York. Su relato "The Bridge Stories: A Short Collection" explora el carácter archipelágico como una poética y como una respuesta simbólica a los legados históricos y políticos del colonialismo extendido, según estos se han venido configurando y experimentando dentro de las particularidades del Caribe insular.

España, Inglaterra, Holanda y Francia controlaron las Islas Vírgenes estadounidenses antes de que éstas se convirtieran en posesión danesa a finales del siglo XVII, por lo que han sido conocidas como las Indias Occidentales Danesas. La isla de St. Croix no era originalmente parte del archipiélago que poseía Dinamarca hasta que Francia la compró en 1733. Desde el 1867, los Estados Unidos trataron de adquirir estas islas, primero como parte del plan del Secretario de Estado William Henry Seward de "expansión territorial pacífica" y luego en un intento de asegurar una ruta hacia el Canal de Panamá para procurar que las islas no cayeran en manos de Alemania durante la primera Guerra Mundial ("Purchase of the United States Virgin Islands, 1917"). ${ }^{12}$ Finalmente, en ${ }^{18}-917$ los Estados Unidos compraron el archipiélago a Dinamarca pagando 25 millones de dólares.

Durante el primer periodo de dominio estadounidense, las fuerzas navales de los EE. UU. administraron estas islas. En 1932 el Departamento del Interior pasó a administrar la región. Inicialmente, los residentes de las islas eran considerados "nacionales" pero no eran ciudadanos estadounidenses. En 1927, el Congreso otorgó la ciudadanía estadounidense a todo el que hubiera nacido en las islas después de 1917. El Acta Orgánica de 1954 definió estas islas como un territorio organizado no incorporado a los Estados Unidos; de ese modo se añadió este territorio a la lista de las posesiones insulares estadounidenses con estatus ambiguo. Los residentes de la isla han elegido a sus propios gobernadores desde el 1970. En 1993 los residentes intentaron celebrar un referéndum sobre el estatus -en el cual los participantes escogieron entre tres opciones: territorio estadounidense, integración

\footnotetext{
${ }^{18}$ Ver los trabajos de William W. Boyer y de Isaac Dookhan sobre la historia de las Islas Vírgenes estadounidenses y en particular sobre su relación con los Estados Unidos (Boyer, 2010; Dookhan, 1974).
} 
a los EE.UU. o independencia-, pero como en esta consulta participaron menos del 30\% de los habitantes de las islas sus resultados no se consideraron válidos (Sekou, 1994). En el 2009, la Quinta Convención Constitucional propuso una constitución para las islas. El Congreso de los EE.UU. la rechazó en el 2010. Este archipiélago colonial es el escenario del relato de Yanique que ejemplifica lo que he denominado como una poética archipelágica.

La estructura de "The Bridge Stores: A short Collection" es la de una serie de viñetas, o estampas, que presentan relatos de las vidas de diversos personajes que existen en un sistema de puentes construido por un artista de las Islas Vírgenes estadounidenses para conectar todo el Caribe. Mi lectura de la colección está inspirada en el análisis que hace Enmanuel Martínez. Este crítico analiza los vínculos entre la antología de Yanique, sus cuentos y una forma archipelágica de escritura (Martínez, 2013). ${ }^{19}$ Mi lectura se detiene en las maneras en que la narradora crea una constelación o una red histórico-espacial y geográfica que caracteriza al archipiélago del Caribe. De tal modo, el relato logra producir una imbricación narrativa de tramas breves en que un pequeño grupo de personajes se relaciona en múltiples planos. Además, argumento que en este texto lo archipelágico funciona simultáneamente como categoría geográfica, como metáfora y como elemento estructural del relato.

La historia transcurre en St. Croix la noche del 3 de julio. Ese día los habitantes de las Islas Vírgenes conmemoran la abolición de la esclavitud, que se produjo en 1848) y la víspera de las fiestas del Día de la Independencia de los EE.UU. Esta coyuntura temporal específica es reveladora, ya que hace referencia al colonialismo extendido de la región al invocar simultáneamente la emancipación de los esclavos negros bajo el gobierno danés y la independencia de la metrópolis estadounidense. La primera estampa comienza con la historia de cierto artista que hace puentes, y quien un día decide realizar su proyecto en un formato más monumental:

Había un constructor de puentes. Hacía puentes que las personas ponían en los lóbulos de las orejas y anillados a sus dedos. Pequeños y diminutos puentes...

Los miembros de su familia insistían en que dejara un legado de verdad. Era famoso por hacer cosas pequeñas. Ellos querían que fuera conocido por grandes obras. Así pues, construyó un puente de verdad. Pagado por los yanquis, aunque no para honrar la memoria [del artista] sino para conveniencia de aquéllos, dicha la verdad [...] Enorme y extendiéndose desde Guyana (el extremo austral del mundo) hasta Miami, que es el extremo septentrional del mundo. Antes de permitir que el público caminara por el puente, [el artista] reunió a toda su familia para sacarse una foto en él. Pero el puente estaba construido como sus otros puentes, que era la única forma en que sabía construirlos: delicado y lindo, pero no resistente al peso.

Cuando la cámara disparó la foto (una luz intensa, hermosa, cegadora) el puente se derrumbó. Y no solamente en ese punto, sino en lugares por todo el Caribe, y así las muchas familias que se habían reunido para sacarse fotos (sin consentimiento expreso) también fueron a parar al océano (Yanique, 2010: 15). ${ }^{20}$

\footnotetext{
${ }^{19}$ Agradezco a Enmanuel Martínez por compartir conmigo este trabajo inédito en 2013.

20 "There was a bridge maker. He made bridges that people put in their earlobes and around their fingers. Tiny little bridges...

His living family insisted that he leave a real legacy. He was famous for small things. They wanted him to be known for big things. So he built a real
}

\section{4}

VISITAS AL PATIO Vol.13, No.2: 22-41, 2019 
En este pasaje, los puentes que construyó el artista vinculan al Caribe insular y continental en un nuevo contexto poscolonial financiado por los intereses estadounidenses. En este texto, el archipiélago aparece como una constelación de territorios insulares y continentales rearticulados por una mirada neoimperial. Y aun así también es redefinido como un archipiélago decolonial gracias a la perspectiva narrativa plurifocal del relato y por el proyecto artístico de los puentes que conectan todas las islas. Esta red de puentes podría leerse como otra iteración del Archipiélago mexicano en el contexto del trabajo de Paredes y de Saldívar sobre el Gran México. También podría leerse como una rearticulación de la Confederación caribeña del XIX del Caribe español o de la Federación de las Indias Occidentales de los 1950 y 1960 del Caribe anglófono. Además, los puentes pueden también referirse a los archipiélagos neocoloniales y transnacionales del siglo XXI. El derrumbe del sistema entero marca la crisis o el final de cierto modelo de organización geopolítica imperial, e insinúa las conexiones simbólicas y culturales que podrían volver a armar el fragmentado archipiélago de forma distinta, es decir, trascendiendo las definiciones de identidad de nación continentales o unitarias.

Las estampas que siguen cuentan las historias de algunos de los otros personajes que están en los puentes cuando colapsa el sistema. Cada historia aparenta ser totalmente independiente, pero de maneras sutiles cada viñeta se imbrica con la anterior y/o la posterior. Por ejemplo, la segunda estampa es la historia de Margo, una mujer oriunda de Dominica. Proviene originalmente de una familia pentecostal, pero se convierte al Islam para casarse con Rashaad, a quien sigue a St. Croix. Años más tarde, su relación entra en crisis y Margo deja a su familia para regresar a Dominica. El 3 de julio, Margo decide regresar a casa para volver a vivir con Rashaad. Esperando a su esposo, Margo decide ir a caminar al puente. Desde allí, piensa que ha visto a su esposo con otra mujer en una embarcación. Desconsolada, decide saltar justo antes de que colapse el sistema entero de puentes.

La tercera estampa narra la crisis de familia entre Salli, Toni y su hijo Pete. Toni es un pescador que en esa época encara la crisis económica de la industria pesquera, precipitada por la construcción del sistema de puentes. Salli decide abandonar a Toni con otro hombre y le confiesa a su hijo Pete que Toni no es su verdadero padre. El 3 de julio, el día antes de que Pete saliera para los EE.UU. para ir a estudiar a un colegio católico, padre e hijo intentan subsanar su relación yéndose de pesca. Al final del día, Pete ve a Margo saltar del puente y muere intentando salvarla.

La cuarta estampa es la historia de Guadalupe, una mujer puertorriqueña cuya familia ha emigrado a St. Croix. ${ }^{21}$ Ella ha decidido competir en el concurso de belleza Srta. Emancipación para que Juan Diego se enamore de ella. Guadalupe representa un palimpsesto de varias referencias a la

bridge. Paid for by the Yankees -not to honor his memory, but really for their own convenience. [...] Huge and stretching from Guyana -the place in the world most south -to Miami- the place in the world most north. Before allowing the public to walk on the bridge he gathered all his family onto it for a picture. But the bridge was built like his others, the only way he knew how, delicate and pretty but not able to bear weight.

When the picture flashed -a big, beautiful, blinding ligh- the bridge fell apart. And not only in that spot but in places all over the Caribbean, so the many families who had gathered to take pictures (without express permission) also went into the ocean" (Yanique, 2010: 15).

${ }^{21}$ Ver los estudios de Mirerza González Vélez y Nadja Ríos Villarini sobre las migraciones puertorriqueñas a St. Croix (González-Vélez, 2014; Ríos Villarini y González-Vélez, 2009-2010). 
marginalidad del Caribe y Puerto Rico en el imaginario colonial de las Islas Vírgenes. Por una parte, los nombres de Guadalupe y de Juan Diego nos remontan al mito de orígenes del culto nacional mexicano a la Virgen de la Guadalupe. Aunque la mención de México-Puerto Rico remite al Archipiélago mexicano que ya he analizado en la sección anterior, en el contexto de esta historia la conexión archipelágica mexicana apunta simbólicamente a las múltiples dimensiones de colonialidad que conforman las relaciones intra-caribeñas. Por otra parte, su plan para recuperar el amor de Juan Diego es muy frágil. El ganar el título de Srta. Emancipación la hace confrontar las divisiones raciales internas entre las personas de piel clara del Caribe español y los de piel oscura de las Indias Occidentales. Guadalupe reconoce que es demasiado blanca para encarnar la emancipación negra en el Caribe anglófono y no logra dejar de sentirse fuera de lugar. Es entonces que decide correr hacia el puente. Allí ve a Margo, a Toni y a Pete:

Había una figura negra, como un saco, que también cruzaba el puente. Había dos figuras en el barco justo abajo. Era tarde y estaba oscuro. La luna estaba alta y era creciente. Quería montarse sobre esa luna. La quería a sus pies, como un barco que la llevara al otro lado de lo que fuera. Era una jodida reina de puros dramones. No sabía qué tenía en común con esas tres figuras. Pero sentía que debía escoger entre un grupo o el otro (Yanique, 2010: 29; las cursivas son mías).

Justo antes de que se desplomara el sistema de puentes, Guadalupe se da cuenta de que ella podría convertirse en el puente humano que conecta esta constelación de historias personales. Y, sin embargo, este personaje es totalmente incapaz de comprender todas las sutilezas y complejidades de su propia intuición. En este preciso instante caen todos los puentes y terminan las narraciones.

El marco archipelágico funciona para articular este cuento en varios órdenes. En primer lugar, el archipiélago es literalmente donde sucede la acción del relato, pero Yanique parece proponer otra definición de archipiélago que transciende a la región del Caribe. Todos los personajes de estas estampas coinciden en St. Croix, pero sus rutas también conectan esta isla con la de Dominica, con países en África y en Asia, con los Estados Unidos, Puerto Rico y con muchos otros lugares en el Caribe. Al mismo tiempo, el archipiélago es un referente a la manera en que cada personaje es imbricado en una red de significados y de malentendidos ocasionados por la yuxtaposición de los diversos hilos narrativos. En el plano estructural, la historia está dividida en narrativas insulares que aparentan no funcionar en conjunto a pesar de que todas están entrelazadas. Las conexiones entre los personajes son muy frágiles, efímeras y aun ilusorias. Se anuncia el momento en que está por cuajar la gran narración, pero esta jamás logra realizarse. Y aun así, esta obra no trata del fracaso de la narrativa archipelágica.

En un orden macroestructural o político, el archipiélago es también la metáfora que articula esta red fallida de puentes. En ese plano, quizás el cuento haga referencia a la fragilidad de la Confederación

\footnotetext{
22 "There was a black sack figure crossing the bridge too. There were two figures in a boat just below. It was late, dark. The moon was high and crescented. She wanted to be on top of that moon. She wanted it at her feet-like a boat to get her across anything. She was such a frigging drama queen. She couldn't know what she had in common with these three figures. But she felt she had to choose one set or the other" (Yanique, 2010: 29).
} 
caribeña, pero muy probablemente lo archipelágico aquí resida en las delicadas redes diaspóricas transnacionales y mundiales que conectan el Caribe colonial y postcolonial del presente con los nodos metropolitanos del Primer Mundo. En el modo narrativo que propone Yanique, el archipiélago decolonial es simultáneamente una fuerza centrípeta y centrífuga, que produce sentidos y sinsentidos, transparencia y opacidad. Es precisamente a través de este gesto ambivalente como "The Bridge Stories" consolidan una teorización alternativa de lo archipelágico que trasciende los legados coloniales del México archipelágico. Esta poética archipelágica alternativa se erige sobre la intuición de que lo que a fin de cuentas conecta las posesiones insulares ultramarinas son precisamente las enormes diferencias que coexisten dentro de una fascinante red de lugares emplazados más allá de los impulsos organizadores de lo imperial, lo nacional o lo continental.

\section{Conclusión: del archipiélago imperial al decolonial}

En los dos casos que he discutido, el Caribe es, de una parte, un archipiélago imperial/colonial imaginado por los principales cartógrafos europeos y los más altos funcionarios españoles de los siglos XVII y XVIII. De otra parte, es un archipiélago decolonial, imaginado, desde abajo, como una constelación o red cultural e histórica de comunidades e individuos. En cada caso, la red de islas y comunidades, vinculadas por las estructuras imperiales y neocoloniales, se reconstituye a sí misma como un sistema de significación que trasciende la organización político-administrativa original del archipiélago.

El archipiélago, entonces, se convierte en una unidad con sentido geográfico capaz de reconectar al Caribe con otros sistemas de islas que han sido imaginadas una y otra vez como archipiélagos coloniales o posesiones ultramarinas de los imperios europeos y del estadounidense. Estos dos casos nos invitan a reflexionar sobre la utilidad de los archipiélagos a la hora de estudiar la especificidad de las experiencias políticas e históricas de regiones insulares que son al mismo tiempo marginales y centrales en los imaginarios imperiales y de la mundialización. Este enfoque comparatista en el estudio de los archipiélagos imperiales, coloniales y decoloniales permite reconectar al Caribe con otras regiones que comparten experiencias semejantes y cuestionan los marcos disciplinares e intelectuales de los estudios regionales, y de las culturas y literaturas nacionales. Quizás ésta sea otra manera de volver a ocuparnos, con intención e inflexión críticas, de las mundializaciones de la primera modernidad y del período contemporáneo. Dentro de esta modalidad de pensamiento, los archipiélagos dejan ya de ser posesiones distantes de ultramar, para transformarse en conjuntos de islas con estados políticos anómalos que desafían la tiranía de los imaginarios tanto nacionales como transnacionales.

\section{BIBLIOGRAFÍA}

“Archipelago". Oxford English Dictionary. Recuperado de: http://www.oed.com/

Arias, S. (2010). Rethinking Space: An Outsider's View of the Spatial Turn. GeoJournal, 75(1), 29-41. 
Balbuena, B. (1971). La grandeza mexicana y Compendio apologético en alabanza de la poesía. México: Porrúa.

Baldacchino, G. (2006). Islands, Island Studies, Island Studies Journal. Island Studies Journal, 1(1), 3-18.

Benítez-Rojo, A. (1996). The Repeating Island. Durham: Duke University Press.

Bjork, K. (1998). The Link that Kept the Philippines Spanish: Mexican Merchant Interests and the Manila Trade, 1571-1815. Journal of World History, 9(1), 25-50.

Blanco, J. (2009). Frontier Constitutions: Christianity and Colonial Empire in the Nineteenth-Century Philippines. Berkeley: University of California Press.

Bonilla, Y. (2012). Non Sovereign Futures? French Caribbean Politics in the Wake of Disenchantment. En: Linden Lewis (Ed.), Caribbean Sovereignty, Democracy and Development in an Age of Globalization (pp. 208-27). New York: Routledge.

Bosch, J. (1981). De Cristóbal Colón a Fidel Castro. El Caribe frontera imperial. Santo Domingo: Alfa y Omega.

Boyer, W. W. (2010). America's Virgin Islands: A History of Human Rights and Wrongs. Durham: Carolina Academic Press.

Colón, C. (1892). Relación del primer viaje de D. Cristóbal Colón para el descubrimiento de las Indias, puesta sumariamente por fray Bartolomé de las Casas. Biblioteca Clásica, 164: Relaciones y cartas de Cristóbal Colón. Madrid: Librería de la viuda de Hernando.

Champlain, S. (1870). Brief Discours des Choses plus remarquables que Sammuel Champlain de Brouage a reconneues aux Indes Occidentalles au voiage qu'il en a faict en icettes en l'année 1599 et en l'année 1601, comme ensuite.

Champlain, S. (1859). Narrative of a Voyage to the West Indies and Mexico, 1599-1602 (London: Printed for the Hakluyt Society.

DeLoughrey, E. M. (2010). Roots and Routes: Navigating Caribbean and Pacific Island Literatures. Honolulu: University of Hawai'i Press.

Dookhan, I. (1974). A History of the Virgin Islands of the United States. Epping: Caribbean Universities Press for the College of the Virgin Islands. 
Flynn, D. O. y A. Giráldez (1995). Born with a "Silver Spoon": The Origin of World Trade in 1571. Journal of World History, 6(2), 201-221.

Frank, G. (1998). ReOrient: Global Economy in the Asian Age. Berkeley: University of California Press.

Fuchs, B. y Y. Martínez-San Miguel (2009). “La grandeza mexicana” de Balbuena y el imaginario de una “metrópolis colonial”. Revista Iberoamericana, 75(228), 675-695.

Fuchs, J. L. (1983). Vincenzo Coronelli and the Organization of Knowledge: The Twilight of Seventeenth-Century Encyclopedism. (Tesis de doctorado). University of Chicago, Chicago.

Glissant, É. (1997). Traité du Tout-monde. Paris: Gallimard.

González Vales, L. (2014) "El situado mexicano y la financiación de las fortificaciones de San Juan de Puerto Rico". [San Juan]: Oficina de Servicios Legislativos de Puerto Rico. Recuperado de http://edicionesdigitales.info/biblioteca/situadomexicano.pdf

González-Vélez, M. (2014). Mapping Points of Origin in the Transnational Caribbean: The Foundational Narrative of the Puerto Rican Pioneer Family in the Virgin Islands. Revista Umbral, $8,46-63$.

Hagimoto, K. (2013). Between Empires: Martí, Rizal and the Intercolonial Alliance. New York: Palgrave.

Harley, J. B. (2001). The New Nature of Maps: Essays in the History of Cartography. Baltimore: Johns Hopkins University Press.

Keegan, W. y M. D. Maclachlan (1989). The Evolution of Avunculocal Chiefdoms: A Reconstruction of Taino Kinship and Politics. American Anthropologist, 91(3), 613-630.

Kincaid, J. (1988). A Small Place. New York: Farrar, Straus, and Giroux.

Las Casas, B. de Las (1988 [1552]). Brevisima relación de la destrucción de las Indias. México: Cátedra Letras Hispánicas.

Limón, J. (1998). American Encounters: Greater México, the United States and the Erotics of Culture. Boston: Beacon Press.

Lockhart, J. y S. B. Schwartz (1989). Early Latin America. New York: Cambridge University Press. 
Martínez, E. (2013). Archipelagic Content and Form in Tiphanie Yanique's The Bridge Stories: A Short Collection. Texto inédito.

Martínez-San Miguel, Y. (2014). Coloniality of Diasporas: Rethinking Intra-colonial Migrations in a Pan Caribbean Context. New York: Palgrave.

Mohammed, P. (2009). Imaging the Caribbean: Culture and Visual Translation. Oxford: Macmillan.

Moll, H. (1701). A System of Geography; or a New \& Accurate Description of the Earth. Tomo 2. London: Timothy Childe.

Moréri, L. (1707). Le Grand Dictionaire Historique. Paris: Denisse Mariette.

Morillo Alicea, J. (2005). Uncharted Landscapes of "Latin America": The Philippines in the Spanish Imperial Archipelago. En: Christopher Schmidt-Nowara y John M. Nieto Phillips. Interpreting Colonialism: Empires, Nations and Legends (pp. 25-53). Albuquerque: University of New Mexico Press.

Mundy, B. E. (1996). The Mapping of New Spain. Chicago: University of Chicago Press.

Nettels, C. (1931). England and the Spanish-American Trade, 1680-1715. Journal of Modern History, 3(1), 1-32.

Paredes, A. (1976). A Texas-Mexican Cancionero. Urbana: University of Illinois Press.

Pedreira, A. S. (1992 [1934]). Insularismo: ensayos de interpretación puertorriqueña. Río Piedras: Edil.

Padrón, R. (2004). The Spacious Word: Cartography, Literature and Empire in Early Modern Spain. Chicago: University of Chicago Press.

Pugh, J. (2013). Island Movements: Thinking with the Archipelago. Island Studies Journal, 8(1), 9-24.

Purchase of the United States Virgin Islands, 1917, U.S. Department of State Archive, January 20, 2001 to January 20, 2009. Recuperado de http://2001-2009.state.gov/r/pa/ho/time/ wwi/107293.htm

Rafael, V. (2005). The Promise of the Foreign: Nationalism and the Technics of Translation in the Spanish Philippines. Durham: Duke University Press. 
Ritchie, C. T. (2014 [1974]). Samuel Champlain. Encyclopedia Britannica. Recuperado de: http:// www.britannica.com/EBchecked/topic/105187/Samuel-de-Champlain

Ríos Villarini, N. y M. González Vélez (2009-2010). Oral Histories of Bilingual Education Teachers from the Puerto Rican Diaspora in St. Croix: Exploring Ideological Tensions Inside and Outside the Classroom. Sargasso, II, 35-49.

Saldívar, J. D. (2012). Trans-Americanity: Subaltern Modernities, Global Coloniality, and the Cultures of Greater Mexico. Durham: Duke University Press.

Schurtz, W. (1959). The Manila Galleon. New York: E. P. Dutton and Company.

Sekou, M. (1994). The Failure of the Political Status Process in the U.S. Virgin Islands. 19 $9^{\text {th }}$ Annual Conference of the Caribbean Studies Association. Mérida, México, 23-27 de mayo.

Stevens-Arroyo, A. (1993). The Inter-Atlantic Paradigm: The Failure of Spanish Medieval Colonization of the Canary and Caribbean Islands. Comparative Studies in Society and History, $35(3), 515-43$.

Stratford, E. et al. (2011). Envisioning the Archipelago. Island Studies Journal, 6(2), 113-30.

Tarver, J. Ch. (1853). Le Dictionnaire Phraséologique Royal: Anglais-Français. London: Dualu \& Co.

Thompson, L. (2010). Imperial Archipelago: Representation and Rule in the Insular Territories under U.S. Dominion after 1898. Honolulu: University of Hawai'i Press.

Wallerstein, I. (1974). The Rise and Future Demise of the World Capitalist System: Concepts for Comparative Analysis. Comparative Studies in Society and History, 16(4), 387-415.

Walton, T. R. (1994). The Spanish Treasure Fleets. Sarasota, FL: Pineapple.

Yanique, T. (2010). The Bridge Stories: A Short Collection. How to Escape a Leper Colony (pp.1530). Minneapolis: Graywolf Press. 